\title{
Erratum to: MIIP remodels Rac1-mediated cytoskeleton structure in suppression of endometrial cancer metastasis
}

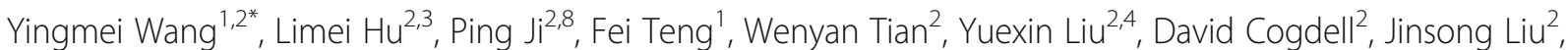
Anil K. Sood ${ }^{5,6}$, Russell Broaddus', Fengxia Xue ${ }^{1 * *}$ and Wei Zhang ${ }^{2,7^{*}+}$

\section{Erratum}

The original article [1] contains an error whereby Fig. 2D mistakenly displays a duplicate image for the panels, 'siMIIP\#1' and 'si-MIIP\#2'.

The correct version of Fig. 2D and its constituent panels can instead be viewed below.
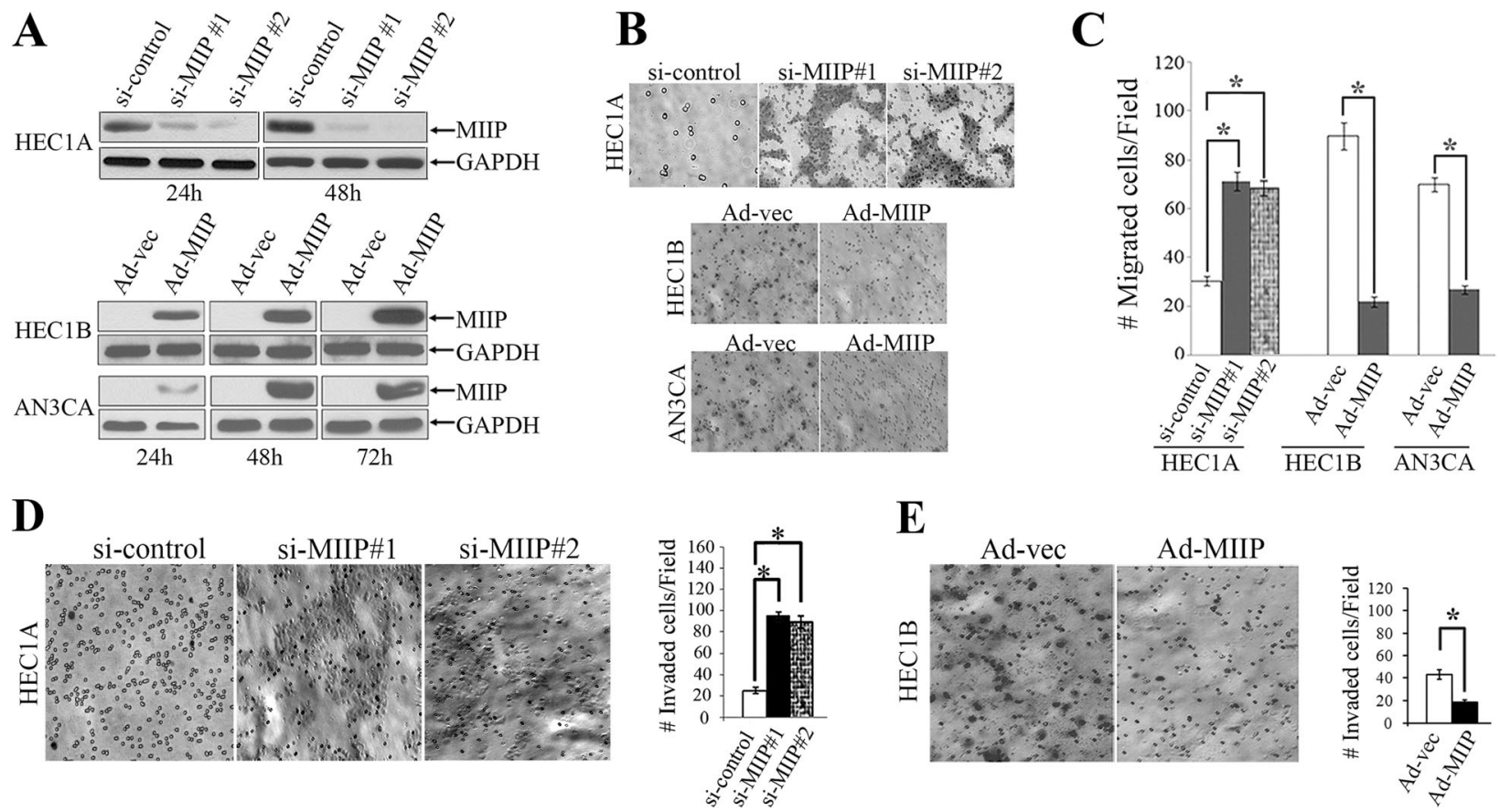

\footnotetext{
*Correspondence: wezhang@wakehealth.edu; fengxiaxue1962@gmail.com;

wangyingmei1978@126.com

${ }^{\dagger}$ Equal contributors

'Department of Gynecology and Obstetrics, Tianjin Medical University General Hospital, Tianjin, China

${ }^{2}$ Department of Pathology, The University of Texas MD Anderson Cancer Center, Houston, TX, USA

Full list of author information is available at the end of the article
} 


\section{Author details}

'Department of Gynecology and Obstetrics, Tianjin Medical University General Hospital, Tianjin, China. ${ }^{2}$ Department of Pathology, The University of Texas MD Anderson Cancer Center, Houston, TX, USA. ${ }^{3}$ Department of Systems Biology, The University of Texas MD Anderson Cancer Center, Houston, TX, USA. ${ }^{4}$ Department of Bioinformatics, The University of Texas MD Anderson Cancer Center, Houston, TX, USA. ${ }^{5}$ Department of Gynecologic Oncology and Reproductive Medicine, The University of Texas MD Anderson Cancer Center, Houston, TX, USA. ${ }^{6}$ Center for RNAi and Non-Coding RNA, The University of Texas MD Anderson Cancer Center, Houston, TX, USA. ${ }^{7}$ Department of Cancer Biology, Comprehensive Cancer Center of Wake Forest Baptist Medical Center, Winston-Salem, NC 27157, USA. ${ }^{8}$ Present Address: Department of Biochemistry and Molecular Biology, The University of Texas Medical Branch, Galveston, TX, USA.

Received: 14 September 2017 Accepted: 14 September 2017

Published online: 06 October 2017

\section{Reference}

1. Wang Y, et al. MIIP remodels Rac1-mediated cytoskeleton structure in suppression of endometrial cancer metastasis. J Hematol Oncol. 2016;9:112. 